\title{
A compact multilayer triplexer using an ultra-wideband diplexer and a low-pass filter for $2.4 \mathrm{GHz}$ wireless systems
}

\author{
Shinpei Oshima ${ }^{1 a)}$, Koji Wada ${ }^{2 b)}$, Ryuji Murata ${ }^{3}$, Hiroshi Hara ${ }^{3 c)}$, \\ Hitoshi Ebihara $^{3}$, and Yukihiro Shimakata ${ }^{3}$ \\ ${ }^{1}$ Department of Electronic Control Engineering, Oyama National College of \\ Technology, 771 Oaza-Nakakuki, Oyama-shi, Tochigi, 323-0806 Japan \\ ${ }^{2}$ Department of Communication Engineering and Informatics, Graduate School of \\ Informatics and Engineering, The University of Electro-Communications, \\ 1-5-1 Chofugaoka, Chofu-shi, Tokyo 182-8585, Japan \\ ${ }^{3}$ TAIYO YUDEN CO., LTD., 8-1, Sakae-cho, Takasaki-shi, Gunma, 370-8522 Japan \\ a)s-oshima@oyama-ct.ac.jp \\ b)wada@ee.uec.ac.jp \\ c) h-hara@jty.yuden.co.jp
}

\begin{abstract}
We propose a triplexer in a low temperature co-fired ceramic substrate for ultra-wideband (UWB) and $2.4 \mathrm{GHz}$ wireless systems. This triplexer can be applied to the band of $2.4 \mathrm{GHz}$ wireless systems $(2.4-2.5 \mathrm{GHz})$, the band group $1(3.168-4.752 \mathrm{GHz})$ of multiband orthogonal frequency-division multiplexing UWB wireless systems, and the band groups 3 and $4(6.336-9.504 \mathrm{GHz})$ of the same systems. The triplexer achieves a compact size $\left(5.3 \times 5.3 \times 0.384 \mathrm{~mm}^{3}\right)$. The insertion losses are less than $2.0 \mathrm{~dB}$ and the isolation characteristics are higher than $24 \mathrm{~dB}$.
\end{abstract}

Keywords: triplexer, low temperature co-fired ceramic (LTCC) substrate, ultra-wideband (UWB), low-pass filter (LPF), UWB diplexer

Classification: Microwave and millimeter wave devices, circuits, and systems

\section{References}

[1] Y.-S. Lin, C.-C. Liu, K.-M. Li, and C. H. Chen, "Design of an LTCC tri-band transceiver module for GPRS mobile applications," IEEE Trans. Microw. Theory Tech., vol. 52, no. 12, pp. 2718-2724, Dec. 2004.

[2] T. Ohno, K. Wada, and O. Hashimoto, "Design methodologies of planar duplexers and triplexers by manipulating attenuation poles," IEEE Trans. Microw. Theory Tech., vol. 53, no. 6, pp. 2088-2095, June 2005.

[3] D. Kim, D. H. Kim, J. I. Ryu, and J. C. Kim, "Highly integrated triplexers for WiMAX applications," IEEE MTT-S Int. Microw. Symp. Dig., pp. 1091-1094, June 2008. 
[4] S. A. Ghorashi, B. Allen, M. Ghavami, and A. H. Aghvami, "An overview of MB-UWB OFDM," IEE Seminar on Ultra Wideband Communications Technologies and System Design, 2004, pp. 107-110, July 2004.

[5] S. Oshima, K. Wada, R. Murata, and Y. Shimakata, "Multilayer dualband bandpass filter in low temperature co-fired ceramic substrate for ultra-wideband applications," IEEE Trans. Microw. Theory Tech., vol. 58, no. 3, pp. 614-623, March 2010.

[6] S. Oshima, K. Wada, R. Murata, and Y. Shimakata, "A study on a multilayer diplexer using LTCC technology for ultra-wideband wireless modules," IEICE Electron. Express, vol. 8, no. 11, pp. 848-853, June 2011.

\section{Introduction}

Compact wireless modules using low temperature co-fired ceramic (LTCC) technology are adapted to a lot of compact wireless systems because some passive components (e.g., bandpass filters, multiplexers and matching circuits) for the module can be built in the LTCC substrate [1]. The multiplexers are very useful components in order to share the antenna of the compact multiband wireless systems $[2,3]$.

Recently, ultra-wideband (UWB) wireless systems are actively researched worldwide [4]. For the compact UWB wireless modules using the LTCC technology, we have studied the wideband bandpass filters [5] and the diplexers with wide passbands [6]. However, there have been few studies about the triplexer for UWB and $2.4 \mathrm{GHz}$ wireless systems using the LTCC technology. In this letter, we present a compact multilayer triplexer for the UWB and $2.4 \mathrm{GHz}$ wireless modules using the LTCC technology. The UWB wireless systems are chosen for the band groups 1, 3, and 4 of the multiband orthogonal frequency-division multiplexing systems [4].

\section{Schematic and simulation}

Figure 1 (a) shows the basic schematic of the triplexer. This triplexer consists of the diplexer for the UWB wireless systems [6], a low-pass filter (LPF) for the $2.4 \mathrm{GHz}$ wireless systems, and matching circuits. The reference impedance of each port is $50 \mathrm{ohm}$. The LPF for the triplexer is required to have good attenuation characteristics and high input impedance from $3.168 \mathrm{GHz}$ to $9.504 \mathrm{GHz}$. In order to achieve the requirements with a compact size, we adapt the LPF which is comprised of inductors and capacitors. Figure 1(b) indicates frequency characteristics of the LPF. It is calculated by a commercial circuit simulator (ADS, Agilent Technologies Inc.). This LPF achieves the good attenuation characteristics and high input impedance at the frequency band of the UWB systems $(3.168-9.504 \mathrm{GHz})$. The diplexer has phase shifter in order to enhance the input impedance at the band of the $2.4 \mathrm{GHz}$ wireless systems. The length of the phase shifter must be chosen, taking into consideration of the good reflection characteristics in the three passbands. 


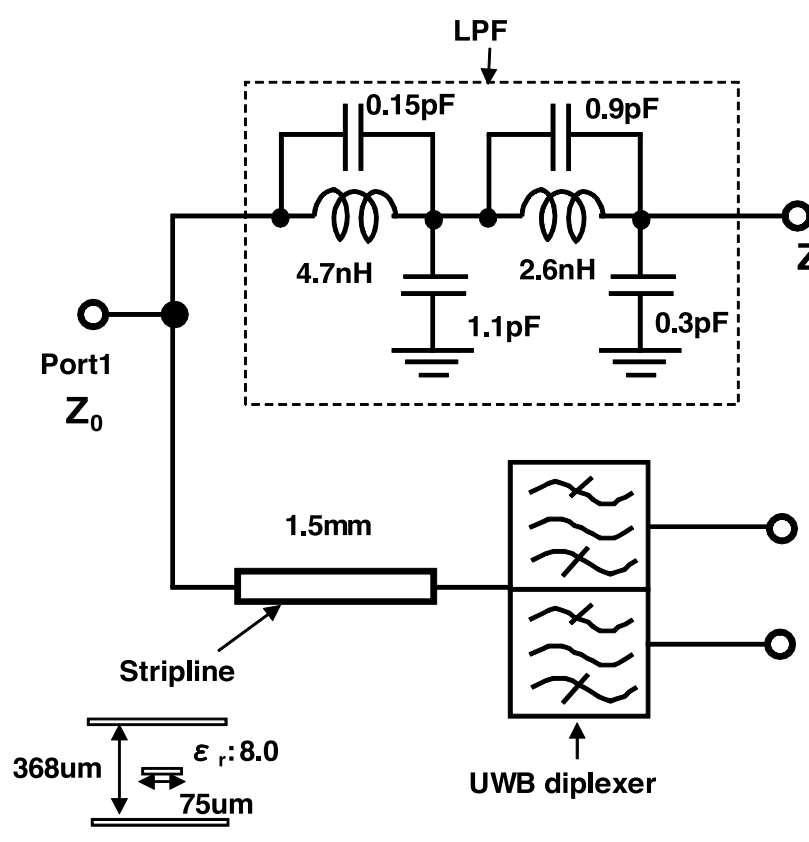

(a)
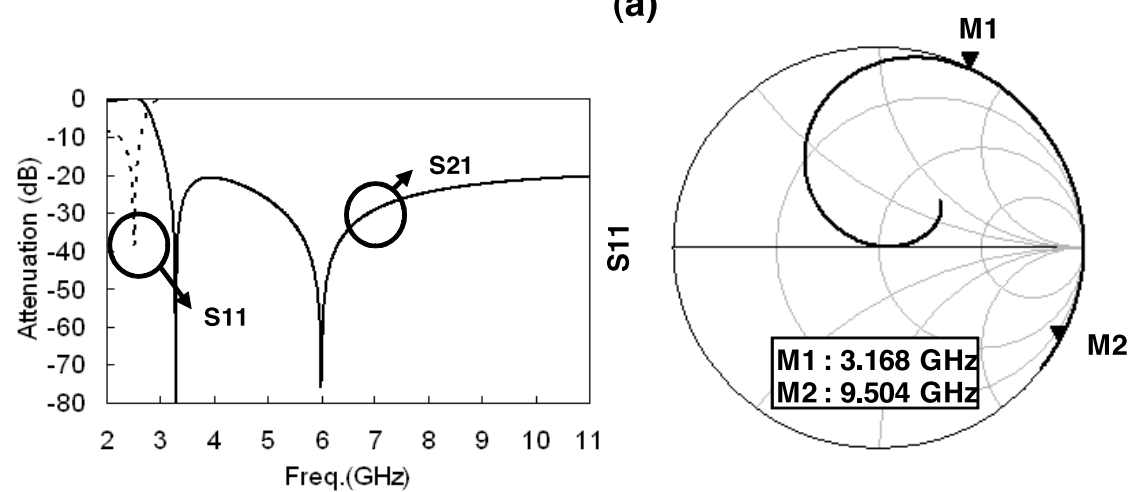

(b)

$2 \mathrm{GHz}-11 \mathrm{GHz}$
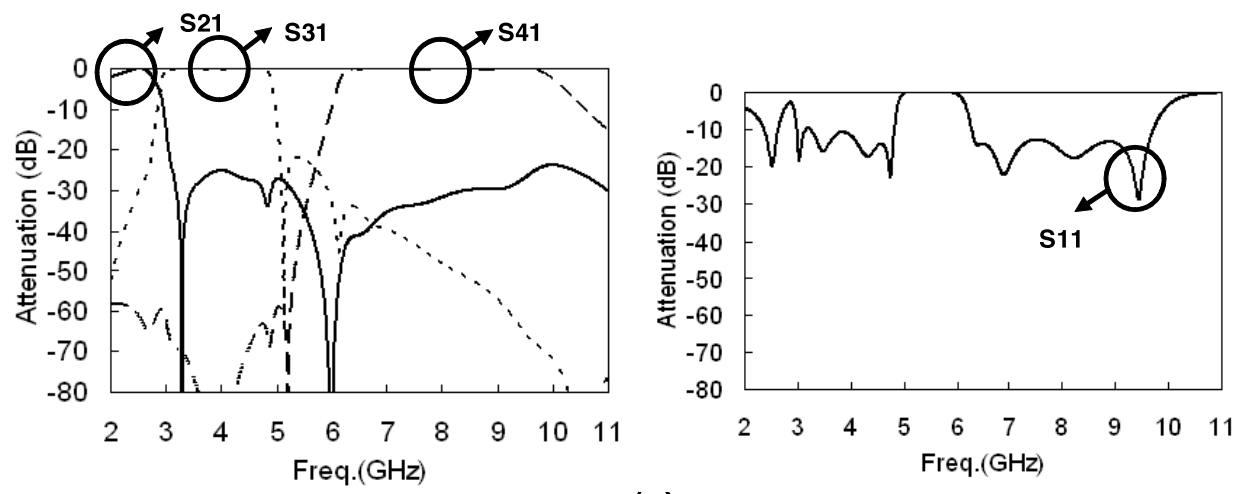

(c)

Fig. 1. (a) Basic schematic of a triplexer, (b) Simulated results of an LPF, (c) Simulated results of a triplexer.

Figure 1 (c) shows the simulated results of the triplexer with the circuit simulator. We confirm that the triplexer separates the bands of the $2.4 \mathrm{GHz}$ wireless systems $(2.4-2.5 \mathrm{GHz})$, the low frequency band of the UWB systems $(3.168-4.752 \mathrm{GHz})$, and the high frequency band of the same systems (6.336 $9.504 \mathrm{GHz})$. 


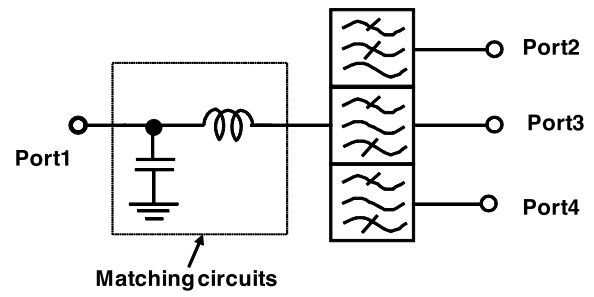

(a)
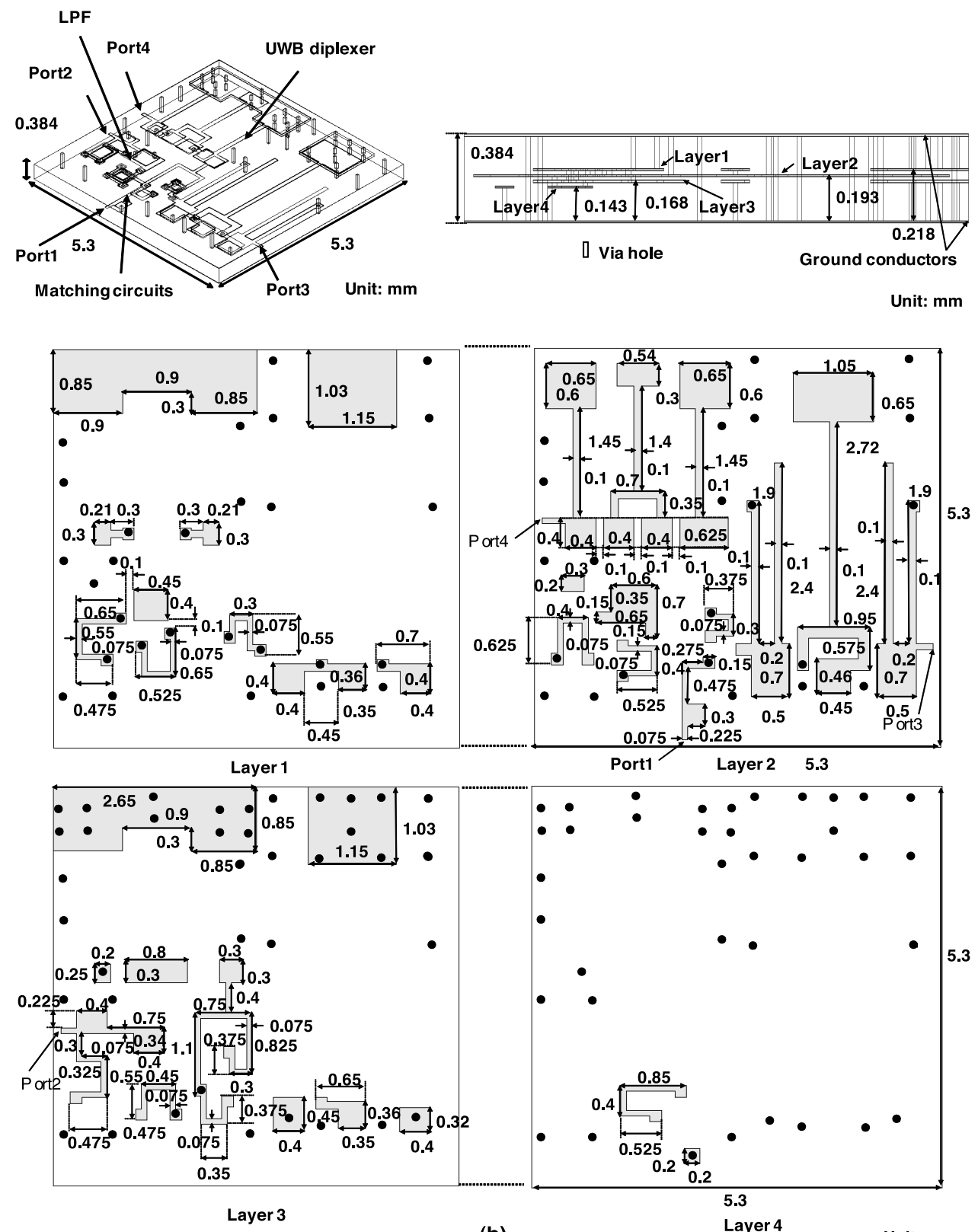

(b)

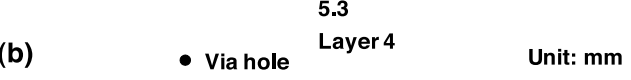

Fig. 2. (a) Schematic of a triplexer with matching circuits at the common port, (b) LTCC structure.

\section{Experiments}

In this section, we design the LTCC structure and fabricate the prototype in order to verify the presented approach. Figure 2 shows the LTCC structure. The triplexer is optimized by means of modifying the structure based on the 


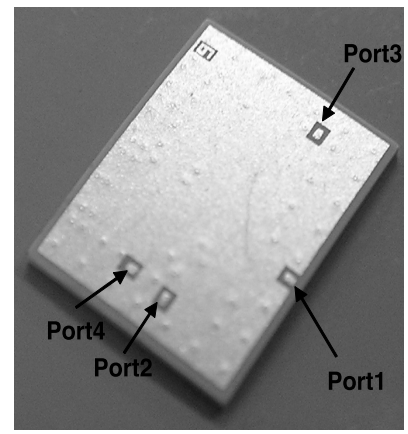

(a)
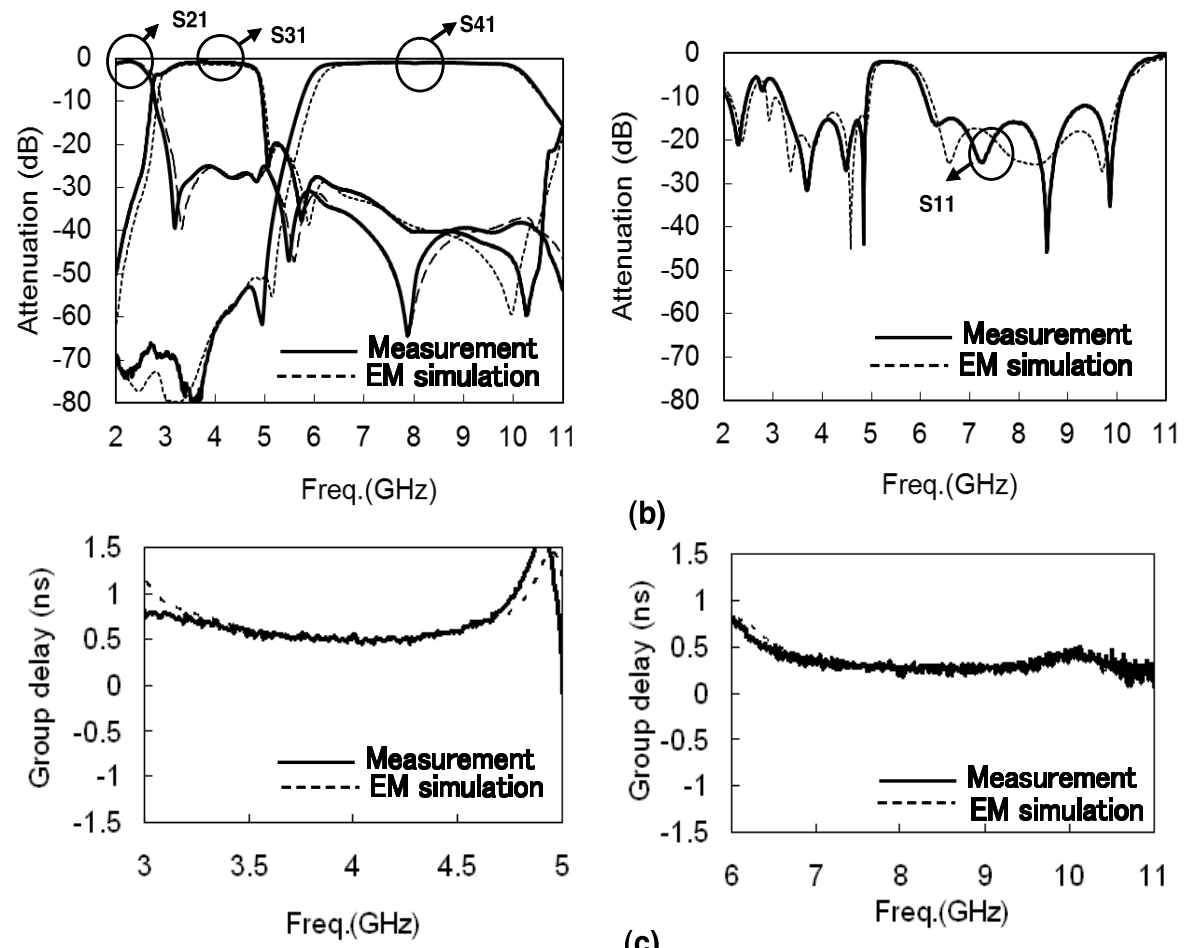

(c)

Fig. 3. (a) Photograph of prototype, (b) Measured results, (c) Measured results of group delays.

basic circuit shown in Fig. 1 (a), taking into consideration of the parasitic effects caused by the three-dimensional LTCC structure. The common port sets the matching circuits as shown in Fig. 2 (a) in order to compensate for the matching conditions due to the parasitic effects of the three-dimensional LTCC structure. The capacitance and inductance of the matching circuits are very small. Figure 2 (b) shows the multilayer structure of the triplexer. The relative permittivity of the LTCC substrate is 8.0. The material of the conductor in the substrate is silver. The thickness of the conductor is $8 \mu \mathrm{m}$. The triplexer consists of the four conductor layers inserted into the middle portion of the LTCC substrate with the ground planes on the top and bottom layers. The dimensions are $5.3 \times 5.3 \times 0.384 \mathrm{~mm}^{3}$.

Figure 3 (a) shows the prototype of the LTCC substrate. The LTCC substrate has electrodes for evaluation using ground-signal-ground (GSG) probes. The LTCC substrate is $7.6 \times 6.0 \times 0.384 \mathrm{~mm}^{3}$ and the compact 
triplexer $\left(5.3 \times 5.3 \times 0.384 \mathrm{~mm}^{3}\right)$ is embedded in this substrate. Figures 3 (b) and (c) show the measured results with simulated results using a commercial electromagnetic simulator (HFSS, ANSYS, Inc.). The prototype is measured with a vector network analyzer (N5230A PNA-L, Agilent Technologies, Inc.) using GSG probes (ACP40, Cascade Microtech, Inc.). The measured results are similar to the simulated results. The insertion losses are less than $2.0 \mathrm{~dB}$ in the three passbands. The isolation characteristics are higher than $24 \mathrm{~dB}$. In the both UWB passbands, the group delays are less than 1 ns. As a result, we can confirm the effectiveness of the presented approach through the experiments.

\section{Conclusion}

A compact multilayer triplexer for the UWB and $2.4 \mathrm{GHz}$ wireless modules is presented. The compact triplexer consists of the UWB diplexer, the LPF for $2.4 \mathrm{GHz}$ wireless systems, and the matching circuits. Simulation and experiments are carried out. The simulated results with EM simulator agree well with experiment. We can achieve the low insertion losses $(2.0 \mathrm{~dB})$ in the three passbands including two UWB passbands and a compact size. In future study, we will try to develop the compact multiband wireless module using the UWB and $2.4 \mathrm{GHz}$ wireless systems. 